\section{The Cognitive Neuroscience of Human Laterality: Lessons From the Bisected Brain}

\author{
Patricia A. Reuter-Lorenz and Andrea C. Miller ${ }^{1}$ \\ Department of Psychology, University of Michigan, Ann Arbor, Michigan
}

With the discovery that surgical separation of the cerebral hemispheres produces two independent and differently abled cognitive spheres, Gazzaniga, Bogen, and Sperry (1962) launched a revolution in the field of psychology: the study of human laterality. More than thirty-five years later, the scientific progeny of Roger Sperry continue to study split-brain patients in order to solve some of the many mysteries confronting cognitive neuroscience today. Historically, split-brain studies catalyzed research on hemispheric differences in neurologically intact subjects. More recently, the bisected brain has served as the proving ground for various models of complex cognitive processes. What lessons have been learned from splitbrain patients? How does research on split-brain patients converge with other methodologies, including the advances in neuroimaging gaining prominence in the field?

\section{Recommended Reading}

Gazzaniga, M.S. (1995). Consciousness and the cerebral hemispheres. In M.S. Gazzaniga (Ed.), The cognitive neurosciences ( $\mathrm{pp}$. 1391-1400). Boston: MIT Press.

Ogden, J.A. (1996). Split brain, split mind? Case L.B. In Fractured minds: A case-study approach to clinical neuropsychology (pp. 232251). New York: Oxford University Press.

Zaidel, E., \& Iacoboni, M. (Eds.). (in press). The role of the corpus callosum in sensory-motor integration: Anatomy, physiology and behavior. New York: Plenum Press.
Our review offers some answers to these questions.

Consisting of more than 200 million nerve fibers, the corpus callosum is the major white-matter pathway connecting the left and right cortical hemispheres. In some individuals with intractable epilepsy, severing this pathway is the medical treatment of choice. Scientists who study the cognitive consequences of the split-brain operation have focused on two major issues. One is to determine what types of mental representations and processes can be shared between the brain's hemispheres in the absence of the corpus callosum. The other is to characterize and compare the competencies of the left and right hemispheres in order to develop and test hypotheses about normal cognitive architecture.

Six patients figure prominently in the corpus of split-brain studies. L.B., N.G., and A.A. are part of the original West Coast series of patients who underwent surgical section of both the corpus callosum and a minor interhemispheric pathway known as the anterior commissure (Bogen \& Vogel, 1975). J.W., V.P., and D.R. are part of the more recent East Coast series for whom only the corpus callosum Baynes, Tramo, \& Gazzaniga, 1992). Each individual has a unique developmental and medical history. Nevertheless, important discoveries resulting from split-brain research converge beautifully with findings from patients with localwas sectioned (callosotomy; e.g., ized "brain damage (due to stroke or other neurological disorders) and research on laterality effects in the intact brain.

Because the brain is organized contralaterally, information from the right visual field (i.e., stimuli on the right side of a visual fixation point; see Fig. 1) travels initially to the left hemisphere of the brain, and information from the left visual field (i.e., stimuli on the left side of a central point) travels initially to the right hemisphere. Both visual laterality studies with neurologically intact participants and split-brain studies require displaying experimental stimuli to the left or right visual field at brief exposure durations that prohibit eye movements (so that the information is truly lateralized to one or the other hemisphere). Performance differences between the hemispheres are typically more robust for split-brain patients than for normal subjects-thus underscoring the importance of the corpus callosum for integrating the operations performed by the cortical hemispheres.

\section{TWO INDEPENDENT COGNITIVE REALMS}

To what extent can the separated hemispheres operate independently? Answers to this question provide insight into the functions of the corpus callosum and the capacities of extracallosal pathways. If the hemispheres still communicate after the corpus callosum has been severed, then patterns of hemispheric asymmetry in split-brain patients should reflect this preserved cross talk.

In fact, the separated hemispheres are virtually isolated from one another, so that one hemisphere is rarely conscious of stimulation presented to the other (Reuter-Lorenz, Nozawa, Gazzaniga, \& 


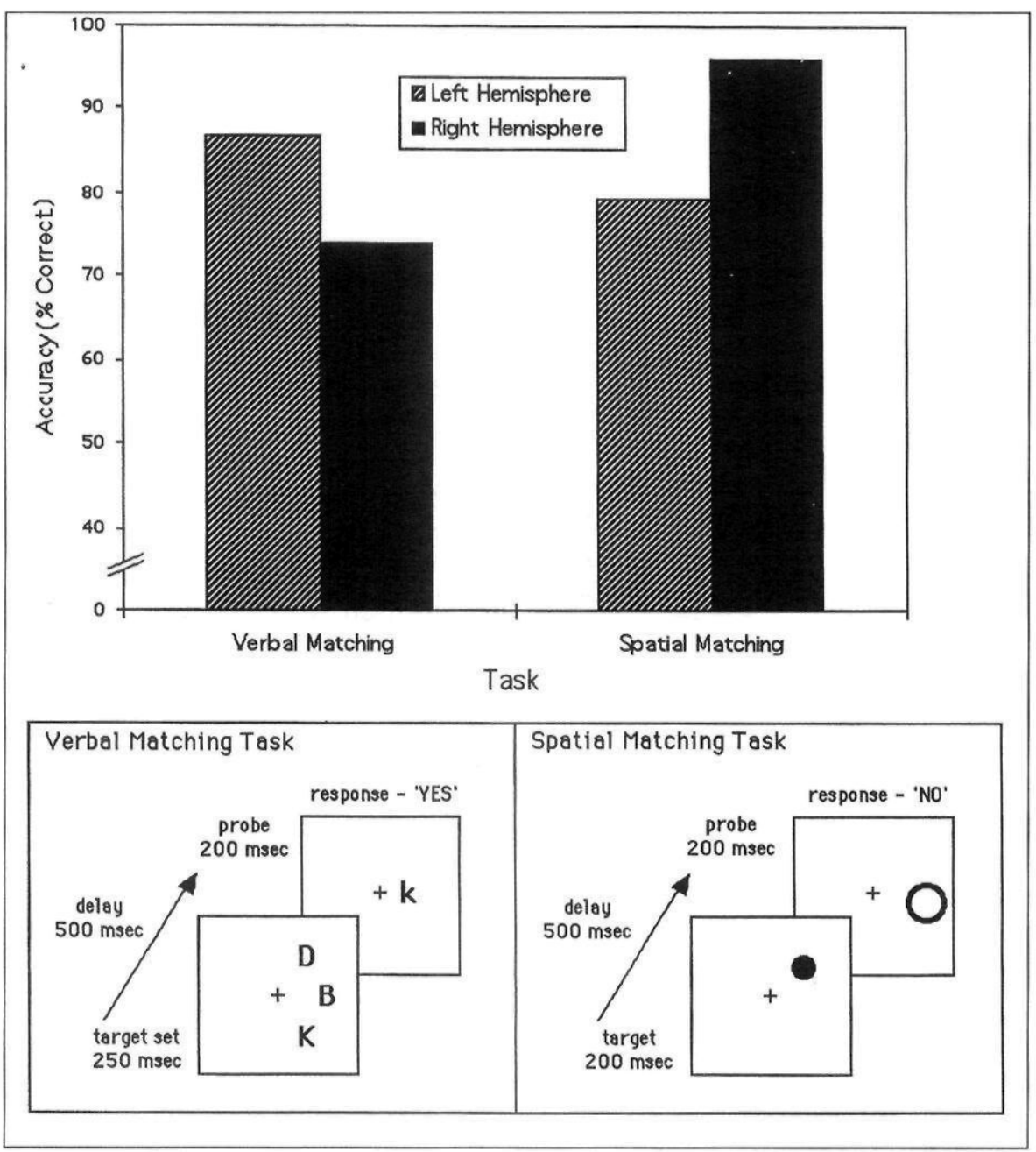

Fig. 1. Accuracy performance of patient V.P. on two simple matching tasks, with an example of a right-visual-field/left-hemisphere trial for each task. During the tasks, V.P. kept her eyes focused on the plus sign at all times.

Hughes, 1995). Visual and tactile information presented to one hemisphere cannot be compared with information presented to the other hemisphere. The separated hemispheres are also unable to share abstract codes or semantic information (Seymour, Reuter-Lorenz, \& Gazzaniga, 1994), despite previous claims to the contrary (Sergent, 1990). Coarsely coded information about spatial location may be shared, but this capacity may be limited to central portions of the visual field (Fendrich, Wessinger, \& Gazzaniga, 1996). Moreover, the separated hemispheres can implement different visuomotor programs concurrently and with minimal interference. For example, callosotomy patients can copy two different shapes, one with the left hand and one with the right, faster and more accurately than normal subjects (Franz, Eliassen, Ivry, \& Gazzaniga, 1996). Thus, the autonomy of the hemispheres produced by callosal section extends beyond perceptual processing into the stages of decision making and response preparation (ReuterLorenz, in press).

With two separate minds in the same cranium, the split-brain patient might seem ripe for interconflict is evident in the early postoperative period. For example, there are reports of one patient who, while grocery shopping, hemispheric conflict. Indeed, such placed items in a cart with one hand but then returned the items to the shelf with the other hand. However, these frankly conflicting responses decrease over time, suggesting the emergence of mechanisms that resolve interhemispheric competition.

In particular, it has been shown that each hemisphere is able to inhibit, or gate, the responses of the other (Reuter-Lorenz et al., 1995; see also Ivry, Franz, Kingstone, \& Johnston, 1998). Because either hemisphere can gate the other's output, the more competent hemisphere can take control of performance on a task (however, see Levy, 1977). Given the vast differences in the processing abilities of the two hemispheres, such flexibility allows split-brain patients to meet the diverse demands of daily life more readily.

\section{COGNITIVE DICHOTOMIES}

Researchers in the field of human laterality have proposed various dichotomies to interpret differences between the two cerebral hemispheres. Of course, no single dichotomy is likely to capture all the important functional asymmetries, especially because many differences between the hemispheres are a matter of degree. Nevertheless, the verbal-spatial and analytic-holistic dichotomies have both figured prominently in theories of laterality. Recent split-brain studies illustrate the value of these distinctions and reveal fundamental asymmetries that any comprehensive theory of human laterality must ultimately explain.

Left-hemisphere specialization for language in right-handed individuals has been recognized since Paul Broca's seminal cases in the mid-19th century. Right-hemisphere specialization for such non- 
verbal functions as spatial processing and face recognition was widely recognized nearly a century later. But it was the pioneering split-brain studies of Sperry, Gazzaniga, and their colleagues that catapulted the verbal-nonverbal dichotomy into mainstream psychology. This dichotomy has been vigorously pursued for decades in visual laterality research, and more recently, in neuroimaging studies of the neurologically intact. The alternative analytic-holistic dichotomy, which was proposed in part to subsume the distinction between verbal and nonverbal processes, also has drawn much inspiration from split-brain research: The left hemisphere perceives the details and features but not the overall configuration, whereas the right hemisphere gets the big picture while missing the details. We next consider each of these dichotomies in turn.

\section{Verbal-Nonverbal Asymmetries}

Hemispheric differences in verbal and spatial abilities are clear. For example, consider the performance of patient V.P. on two simple matching tasks. We have obtained these data as part of a large-scale investigation on working memory in the separated hemispheres (unpublished observations). In the verbal task, a set of two, three, or four target letters is flashed briefly to the left or right visual field, followed by a probe letter in the same visual field. V.P. then indicates whether or not the probe letter is the same as one of the targets. In the spatial task, a dot is presented briefly in the left or right visual field, followed by a probe circle. V.P. then indicates whether or not the probe is in the same location as the target dot. Figure 1 illustrates these tasks and presents results showing that with a 500-ms delay between the target and probe, the left hemisphere is clearly superior in the verbal task, whereas the right hemisphere excels at the spatial task. Moreover, we find that the magnitude of asymmetry increases as the delay between the target and probe increases to $3 \mathrm{~s}$. These results indicate that the hemispheres are differentially specialized for verbal and spatial representations. The increased asymmetry with delay may stem partly from hemispheric differences in the quality of these initial representations. However, the processes that maintain verbal and spatial representations in working memory may also be differentially lateralized in the left and right hemispheres (e.g., Smith \& Jonides, 1997).

Working memory asymmetries underscore a crucial dimension of the verbal-nonverbal dichotomy: Its explanatory power lies in an emphasis on the relative competence of each hemisphere for engaging verbal versus nonverbalspatial processing operations. This point is further evident from tasks that use letters as stimuli but reveal a right-hemisphere superiority. For example, the right hemisphere of patient L.B. is better than the left when matching letter shapes or mentally rotating letters, even though the left hemisphere is superior at discriminating the letters' identities (Corballis \& Sergent, 1989; Eviatar \& Zaidel, 1994).

Although verbal and nonverbal asymmetries do exist, not all hemispheric differences are accurately described by this dichotomy. The left hemisphere is superior to the right in some abilities that are not obviously linguistic, such as making skilled hand movements, coding temporal sequences, and analyzing certain types of spatial relations (e.g., Kosslyn \& Koenig, 1992). Furthermore, the right hemisphere excels with certain figurative and connotative aspects of language (see Beeman \& Chiarello, this issue). Because one goal of laterality research is to specify fundamental processing differences between the two hemispheres, researchers have attempted to go beyond the verbal-nonverbal dichotomy.

\section{Analytic Versus Holistic Processing}

The most prominent alternative to the verbal-nonverbal dichotomy involves the distinction between analytic and holistic processing. According to this distinction, the left hemisphere is specialized for sequential analysis of features and detailed elements that make up complex stimuli, whereas the right hemisphere is specialized for simultaneous, gestalt-type processing that encodes elements in parallel as a synthesized whole. This distinction was prompted by early evidence from split-brain patients indicating right-hemisphere dominance for recognizing faces and processing configurations versus left-hemisphere superiority for analyzing features and details (e.g., Levy, 1977).

New and striking results supporting this distinction continue to emerge from split-brain research. In a recent study, Gazzaniga (interviewed in Singer, Chedd, \& Angier, 1997) tested J.W.'s perception of paintings of faces made from configurations of objects, such as fruits. When a painting was presented to his right hemisphere, J.W. perceived it as a face, but when the same stimulus was presented to his left hemisphere, he perceived it only as fruits. Numerous other studies on face perception in splitbrain patients confirm a righthemisphere superiority for faces and other complex, hard-to-name forms (see, e.g., Stone, Nisenson, Eliassen, \& Gazzaniga, 1996). The right hemisphere's disadvantage at analyzing elementary features is 
evident in a different line of research on visual imagery. Kosslyn and his colleagues showed that J.W.'s right hemisphere has a specific deficit in imagining the parts of complex forms, whereas his left hemisphere's ability to imagine such parts produces an advantage on a variety of imagery tasks (e.g., Kosslyn \& Koenig, 1992).

Other attempts to investigate the analytic-holistic distinction have yielded mixed results, however. Hierarchical stimulus configurations have been particularly popular for testing the dichotomy. These stimuli consist of large letters formed from appropriate arrangements of smaller letters (e.g., a large $H$ made up of small $S s$ ), representing the global and local levels, respectively. Robertson and her colleagues (see, e.g., Robertson, Lamb, \& Zaidel, 1993) have shown that localized right-hemisphere damage impairs processing at the global level, as the holistic characterization of right-hemisphere processing would predict. Likewise, localized left-hemisphere damage impairs local-level processing, as would be expected if the left hemisphere is specialized for analyzing parts of complex configurations. However, some visual laterality studies of neurologically intact observers have not found global-local processing asymmetries (see Van Kleeck, 1989, for a review). Similarly, although Robertson et al. (1993) reported a left-hemisphere advantage for local processing and a right-hemisphere advantage for global processing for patients L.B., N.G., and A.A., a follow-up study by Weekes, Carusi, and Zaidel (1997) found a global-processing advantage for both hemispheres of these patients.

It is important to recognize that the logic of inferring a general, fundamental processing asymmetry from hemispheric differences on specific tasks such as face recognition and letter identification may be fundamentally flawed. Perhaps the subsystems that process faces and visual language are domainspecific (Moscovitch, Winocur, \& Behrmann, 1997; Polk \& Farah, 1998). That is, these processors may be activated exclusively by particular classes of stimuli. If a processing subsystem in the right hemisphere is specialized for faces, it may indeed process facial features and their relations in a gestalt fashion. However, to the extent that this "module" is domain-specific, it may not be more generally applicable to processing any other gestalt-like configurations.

\section{THE BISECTED BRAIN IN COGNITIVE NEUROSCIENCE}

Split-brain studies have played a vital role in unearthing basic principles governing interhemispheric interactions and human laterality. Here, we survey three important ways in which split-brain research will continue to play a vital role in the field of cognitive neuroscience.

\section{Convergence With Lesion Studies and \\ Functional Neuroimaging}

Because cognitive neuroscience is a field of diverse methodologies, results that converge on the same conclusion provide powerful evidence. Numerous examples of convergence exist among results from split-brain patients, studies of patients with localized brain damage, and functional neuroimaging. Several of these examples are particularly revealing about the unique status of evidence from split-brain patients.

For example, studies of singleword reading in the separated hemispheres of J.W. (ReuterLorenz \& Baynes, 1992) help to show how the isolated language- dominant and -nondominant hemispheres read. Not surprisingly, J.W.'s left hemisphere reads normally. In contrast, his right hemisphere uses a serial, letter-byletter strategy resembling that found in patients with pure alexia (a reading disorder that is acquired in adulthood and results from damage to left occipital-temporal regions of the brain). Processing of single letters' identities by J.W.'s right hemisphere is also impaired; this deficit is like that shown by pure alexics. In pure alexia and in the isolated right hemisphere, a plodding letter-by-letter strategy results because letter identities cannot be accessed efficiently and automatically, as they are in normal reading.

These results have helped resolve a prevailing debate. Previously, it was unclear whether the letter-by-letter strategy seen in pure alexia reflects the limited reading competence of the intact right hemisphere or the impaired reading of the dysfunctional left hemisphere. By demonstrating letter-by-letter reading in the right hemisphere of a split-brain patient, this work suggests that letter-byletter reading in pure alexics reflects reliance on the right hemisphere rather than on the impaired left hemisphere. These conclusions converge with neuroimaging evidence for a left-hemisphere letterprocessing module in the intact brain (Petersen, Fox, Snyder, \& Raichle, 1990; Polk \& Farah, 1998). In this example, a split-brain study illustrates the cognitive consequences (i.e., letter-by-letter reading) associated with the relative absence of a particular processing subsystem, namely, the letteridentity processor.

\section{Revealing the Modules of Cognition}

By characterizing the competencies of the separated hemispheres, 
the cognitive neuroscientist gains insight about which cognitive operations can be dissociated. We summarize two examples to illustrate this point. Studies of the bisected brain, patients with localized brain damage, and neuroimaging evidence (see Mangun et al., 1994) reveal that the right hemisphere has a special role in allocating attention to spatial locations. In contrast, recent evidence indicates that the left hemisphere is essential for attending to objects. For example, one study examined J.W.'s speed in responding to a visual target following a cue. J.W.'s left hemisphere responded more quickly when the cue and target appeared in different locations within the same object than when they appeared in two different objects. In contrast, the right hemisphere did not show this withinobject advantage, suggesting that it orients attention to locations on a purely spatial basis, without reference to their object groupings (Egly, Rafal, Driver, \& Starrveveld, 1994). These results are revealing about the functional architecture of the attention system. They favor the view that object-based attention is mediated by a subsystem that is separable from right-hemisphere attention processes.

The recent case of left-handed split-brain patient V.J. is particularly fascinating. V.J.'s left hemisphere can speak, read, and spell, but it cannot write. Instead, her right hemisphere controls the motor output for writing, even though it cannot speak, read aloud, or spell (Baynes \& Eliassen, 1998). Reading and writing are known to be mediated by dissociable mechanisms, because acquired disorders of reading and writing do not always co-occur in the same patient. However, V.J. raises the more remarkable possibility that in at least some left-handers, writing skills can be localized in the right hemisphere while other major linguistic pro- cesses can be controlled by the language-rich left hemisphere. Evidence such as this will surely stimulate neuroimaging studies of the left-handed brain to establish the generality of this dissociation, and may prompt a reconsideration of processing models of written language in which graphic functions depend on other language processes.

\section{Functional Architecture and Language Competence}

Even in right-handed split-brain patients, there is clear variability in the language competence of the isolated right hemisphere, and, in some cases, speech abilities emerge in the right hemisphere many months or years after surgery (Baynes \& Eliassen, 1998). Such plasticity in the adult brain is a reminder that despite lateralization of function, redundancy also exists, sometimes in the form of latent potential.

Nevertheless, the linguistic superiority of the left hemisphere has fascinating and diverse cognitive consequences. Gazzaniga (1995) refers to the capacity that emerges from the language-rich left hemisphere as the interpreter. The interpreter is the source of the ongoing, conscious narrative that endeavors to explain (often erroneously) the cognitions, emotions, and behaviors produced by nonverbal processing, including that of the right hemisphere.

The cognitive ramifications of the left-brain interpreter have recently been explored in the domain of memory. Metcalfe, Funnell, and Gazzaniga (1995) showed that the right hemisphere is superior at remembering the veridical content of previously presented materials. The left hemisphere, in contrast, is more likely to elaborate and make inferences based on what it sees and, consequently, has more diffi- culty distinguishing what was actually seen from what was merely inferred (see also Phelps \& Gazzaniga, 1992; however, see Beeman \& Chiarello, this issue, regarding right-hemisphere inference processing with an intact corpus callosum). Miller, Kingstone, and Gazzaniga (1997) recently extended this evidence by showing that the left hemisphere is better than the right at using elaborative encoding strategies. The left hemisphere readily uses the meaning or semantic category of to-be-remembered items to form a richer and more durable memory trace. Although the right hemisphere is able to categorize items, it does not utilize these semantic associations in the memory-encoding process. Thus, studies of the bisected brain indicate the lateralization of particular specialized modular abilities while revealing the importance of such modules for more complex cognitive capacities.

\section{CONCLUSION}

As neuroscience enters a new era in the study of brain-behavior relationships, research on the bisected brain will continue to be a major theoretical and empirical force. For decades, methodological and technical limitations forced a reliance on the hemisphere as the primary unit of analysis in neuropsychology. With the advent of neuroimaging techniques, researchers can now draw conclusions about the precise functions of structurally defined brain regions. But knowing the precise locus of a process in the brain still leaves many questions unanswered. Valuable insights into the role of these processes within the overall cognitive architecture can be gleaned from specifying the cognitive capabilities and limitations of the separated hemispheres in the bisected brain. 
Acknowledgments-This work was - supported by a Rackham Faculty Fellowship to P.R.-L. and by a National Science Foundation Graduate Fellowship to A.M. The authors are grateful to David E. Meyer for his thoughtful comments on an earlier version of this manuscript.

\section{Note}

1. Address correspondence to Patricia A. Reuter-Lorenz, Department of Psychology, University of Michigan, 525 East University, Ann Arbor, MI 48109-1109; e-mail: parl@umich.edu.

\section{References}

Baynes, K., \& Eliassen, J.C. (1998). The visual lexicon: Its access and organization in commissurotomy patients. In M. Beeman \& C. Chiarello (Eds.), Right hemisphere language comprehension: Perspectives from cognitive neuroscience (pp. 79104). Mahwah, NJ: Erlbaum.

Baynes, K., Tramo, M.J., \& Gazzaniga, M.S. (1992). Reading with a limited lexicon in the right hemisphere of a callosotomy patient. Neuropsychologia, 30, 187-200.

Bogen, J.E., \& Vogel, P.J. (1975). Neurological status in the long term following cerebral commissurotomy. In F. Michel \& B. Schott (Eds.), Les syndromes de disconnexion calleause chez l'homme (pp. 227-251). Lyon, France: Hospital Neurologique.

Corballis, M.C., \& Sergent, J. (1989). Mental rotation in a commissurotomized subject. Neuropsychologia, 27, 585-597.

Egly, R., Rafal, R., Driver, J., \& Starrveveld, Y. (1994). Covert orienting in the split brain reveals hemispheric specialization for objectbased attention. Psychological Science, 5, 380383.

Eviatar, Z., \& Zaidel, E. (1994). Letter matching within and between the disconnected hemispheres. Brain and Cognition, 25, 128-137.
Fendrich, R., Wessinger, C.M., \& Gazzaniga, M.S. (1996). Nasotemporal overlap at the retina vertical meridian: Investigations with a callosotomy patient. Neuropsychologia, 34, 637-646.

Franz, E.A., Eliassen, J.C., Ivry, R.B., \& Gazzaniga, M.S. (1996). Dissociation of spatial and temporal coupling in the bimanual movements of callosotomy patients. Psychological Science, 7, 306-310.

Gazzaniga, M.S. (1995). Principles of human brain organization derived from split-brain studies. Neuron, 14, 217-218.

Gazzaniga, M.S., Bogen, J.E., \& Sperry, R.W. (1962). Some functional effects of sectioning the cerebral commissures in man. Proceedings of the National Academy of Sciences, USA, 48, 1765-1769.

Ivry, R.B., Franz, E.A., Kingstone, A., \& Johnston, J.C. (1998). The psychological refractory period effect following callosotomy: Uncoupling of lateralized response codes. Journal of Experimental Psychology: Human Perception and Performance, 24, 463-480.

Kosslyn, S.M., \& Koenig, O. (1992). Wet mind: The new cognitive neuroscience. New York: Free Press.

Levy, J. (1977). Manifestations and implications of shifting hemi-inattention in commissurotomy patients. In E.A. Weinstein \& R.P. Friedland (Eds.), Advances in neurology (Vol. 18, pp. 3339). New York: Raven Press.

Mangun, G.R., Hillyard, S.A., Luck, S.J., Handy, T., Plager, R., Clark, V.P., Loftus, W., \& Gazzaniga, M.S. (1994). Monitoring the visual world: Hemispheric asymmetries and subcortical processes in attention. Journal of Cognitive Neuroscience, 6, 267-275.

Metcalfe, J., Funnell, M., \& Gazzaniga, M.S. (1995). Right-hemisphere memory superiority: Studies of a split-brain patient. Psychological Science, 6, 157-164.

Miller, M.B., Kingstone, A., \& Gazzaniga, M.S. (1997). HERA and the split-brain. Society for Neuroscience Abstracts, 22, 1579

Moscovitch, M., Winocur, G., \& Behrmann, M. (1997). What is special about face recognition? Nineteen experiments on a person with visual object agnosia and dyslexia but normal face recognition. Journal of Cognitive Neuroscience, 9 , 555-604.

Petersen, S.E., Fox, P.T., Snyder, A.Z., \& Raichle, M.E. (1990). Activation of extrastriate and frontal cortical areas by visual words and word-like stimuli. Science, 249, 1041-1044.

Phelps, E.A., \& Gazzaniga, M.S. (1992). Hemi- spheric differences in mnemonic processing: The effects of left hemisphere interpretation. Neuropsychologia, 30, 293-297.

Polk, T.A., \& Farah, M.J. (1998). The neural development and organization of letter recognition: Evidence from functional neuroimaging, computational modeling, and behavioral studies. Proceedings of the National Academy of Sciences, USA, 95, 847-852.

Reuter-Lorenz, P.A. (in press). Attention and the bisected brain: Implications for an analysis of callosal function. In E. Zaidel \& M. Iacoboni (Eds.), The role of the corpus callosum in sensorymotor integration: Anatomy, physiology and behavior. New York: Plenum Press.

Reuter-Lorenz, P.A., \& Baynes, K. (1992). Modes of lexical access in the callosotomized brain. Journal of Cognitive Neuroscience, 4, 155-164.

Reuter-Lorenz, P.A., Nozawa, G., Gazzaniga, M.S., \& Hughes, H.C. (1995). The fate of neglected targets in the callosotomized brain: A chronometric analysis. Journal of Experimental Psychology: Human Perception and Performance, 21, 211230.

Robertson, L.C., Lamb, M.R., \& Zaidel, E. (1993). Interhemispheric relations in processing hierarchical patterns: Evidence from normal and commissurotomized subjects. Neuropsychology, 7, 325-342.

Sergent, J. (1990). Furtive incursions into bicameral minds. Integrative and coordinating role of subcortical structures. Brain, 113, 537-568.

Seymour, S., Reuter-Lorenz, P.A., \& Gazzaniga, M.S. (1994). The disconnection syndrome: Basic findings reaffirmed. Brain, 117, 105-115.

Singer, J., Chedd, G., \& Angier, J. (1997). Pieces of mind: Inside the human body [Videotape]. (Available from Connecticut Public Television, Hartford, CT)

Smith, E.E., \& Jonides, J. (1997). Working memory: A view from neuroimaging. Cognitive Psychology, 33, 5-42.

Stone, V.E., Nisenson, L., Eliassen, J.C., \& Gazzaniga, M.S. (1996). Left hemisphere representations of emotional facial expressions. Neuropsychologia, 34, 23-29.

Van Kleeck, M. (1989). Hemisphere differences in global versus local processing of hierarchical visual stimuli by normal subjects: New data and a meta-analysis of previous studies. Neuropsychologia, 27, 1165-1178.

Weekes, N.Y., Carusi, D., \& Zaidel, E. (1997). Interhemispheric relations in hierarchical perception: A second look. Neuropsychologia, 35, $37-44$. 
This document is a scanned copy of a printed document. No warranty is given about the accuracy of the copy. Users should refer to the original published version of the material. 